\title{
JMSR
}

Journal of Medical and Scientific Research

\section{A case of congenital diaphragmatic hernia with malrotation of gut, delayed presentation in adult}

\author{
Sai Mounica Pitchika ${ }^{1}$, Poornima Chowdary DB ${ }^{1}$ and B V Prasad Mallipudi ${ }^{1, *}$
}

${ }^{1}$ Department of Surgical Gastroenterology, Krishna Institute of Medical Sciences, Minister Road, Secunderabad-500003, Telangana, India.

\begin{abstract}
Congenital diaphragmatic hernia (CDH), which mainly occurs in the new born or in childhood with severe respiratory distress and is associated with high mortality, is rarely found in adult. Pulmonary hypoplasia and pulmonary hypertension contribute to the high mortality and morbidity due to CDH. These patients adjust their lifestyle to manage symptoms associated with frank herniation of the large bowel, omentum, and other viscera inside the diaphragmatic hernia. We report a 25 -year-old male who presented with shortness of breath on exertion diagnosed as having CDH. The disease was detected using chest X-ray (CXR) and was confirmed on contrast enhanced CT scan. A defect was noted in the posterolateral right diaphragm with omentum, small bowel, caecum, appendix, and colon herniating through it with malrotation (incomplete rotation) of gut. CT scan and CXR were the two useful methods in diagnosis of CDH in this patient, but sometimes becomes challenging because of rare incidence and types prior to surgery. For better outcome of CDH patients long term follow up and more investigations are needed. In some cases, where conventional CT scan and ultrasonography fails to diagnose a case of $\mathrm{CDH} \&$ malrotation of gut, diagnostic laparoscopy plays a key role in facing such situations.
\end{abstract}

Keywords: diaphragmatic hernia; malrotation of gut;respiratory distress; diagnostic laparoscopy

*Corresponding author: Dr. Mallipudi B V Prasad, M.S, FRCS (Ire), FRCS (Eng)., Senior Consultant \& HOD, Department of Surgical Gastroenterology, Krishna Institute of Medical Sciences, Minister Road, Secunderabad-500003, Telangana, India. Email: prasad@mallipudi.com.

Received 5 July 2021; Revised 18 August 2021; Accepted 24 August 2021; Published 2 September 2021

Citation: Pitchika SM, Chowdary DBP, Mallipudi BVP. A case of congenital diaphragmatic hernia with malrotation of gut, delayed presentation in adult. J Med Sci Res. 2021; 9(4):228-232. DOI: http://dx.doi.org/10.17727/JMSR.2021/9-35

Copyright: (C) 2021 Pitchika SM et al. Published by KIMS Foundation and Research Center. This is an open-access article distributed under the terms of the Creative Commons Attribution License, which permits unrestricted use, distribution, and reproduction in any medium, provided the original author and source are credited.

\section{Introduction}

Congenital diaphragmatic hernia ( $\mathrm{CDH})$ occurs due to incomplete muscularization of the diaphragm. Incidence of $\mathrm{CDH}$ is 1:2500 to 1:3000 live births, and increase of mortality when pulmonary hypoplasia and pulmonary hypertension are associated with $\mathrm{CDH}$ [1]. In $15-20 \%$ of cases with $\mathrm{CDH}$, the hernia occurs on the right side and in $80-85 \%$ of subjects on the left side. Because of protective effect of liver on right side, herniation of liver and colon are extremely rare [2]. The diaphragm is rarely affected on both sides $[1,3]$. The survival of these patients is estimated to be $55-65 \%$ [4].

$\mathrm{CDH}$ includes Bochdalek hernia (70\%) in the posterior-lateral and Morgagni hernia (25-35\%) in 
the anterior or central (2-5\%) part of the diaphragm [5]. Despite the high prevalence of Bochdalek hernia during infancy, the disease is rare in adults and the diagnosis of this type of hernia is very difficult and, in most patients, it is not diagnosed due to the mild delayed manifestation of CDH. Patients with delayed manifestation of $\mathrm{CDH}$ have better prognosis than patients with early manifestation. Small intestine finds a way into thoracic hernia more than any other abdominal organs [2]. The most common clinical manifestation in infants is respiratory distress while, in adults, mild respiratory and gastrointestinal symptoms are more prevalent, and $25 \%$ of the hernia is asymptomatic [6]. Respiratory symptoms are prominent in the right hernia while left hernia shows itself by gastrointestinal symptoms. Moreover, the short-term pulmonary results of patients with right $\mathrm{CDH}$ are not worse than those of patients with left $\mathrm{CDH}$ [1]. In this case study, we have reported an adult patient with left congenital diaphragmatic hernia, presenting with respiratory symptoms.

Intestinal malrotation is also a paediatric condition with most (90\%) of cases diagnosed in the first year of life. Its incidence in childbirths is 1:6000. The incidence in adults seems to be increasing with the higher use of diagnostic imaging exams. Physiologic herniation of the midgut through the umbilical cord occurs during the fourth to fifth week of gestation, and through to the ninth to tenth week when it returns to the abdominal cavity attached to the retroperitoneum. During this process, a $270^{\circ}$ anticlockwise rotation around the upper mesenteric artery occurs, resulting in duodenal arch formation, later emerging as the Treitz ligament (TL). The duodenojejunal loop shifts to the left, and the distal small bowel shifts progressively to the right; the cecum descent completes the rotational process. Finally, themesenteryadheresto the retroperitoneum diagonally from the TL to the cecum.

In this case study, we reported an adult patient with incomplete rotation of gut presenting incidentally at the time of repair of $\mathrm{CDH}$.

\section{Case presentation}

25-year-old male patient presented with shortness of breath on exertion for 4 months. His bowel habits were normal. On examination, air entry was diminished on the left side. Bowel sounds were heard over left hemithorax. Cardiac examination was normal. His abdomen was scaphoid, non-tender, and soft. Chest radiograph showed features of diaphragmatic hernia and hyperlucency of the right lower lobe of lung.

To confirm the diagnosis and have precise knowledge of the herniating viscera, axial cross-sectional chest CT scan was done with intravenous and oral contrast. This confirmed CDH in the left hemi diaphragm with omentum, small bowel, and colon herniating into thoracic cavity (Figures 1, 2).

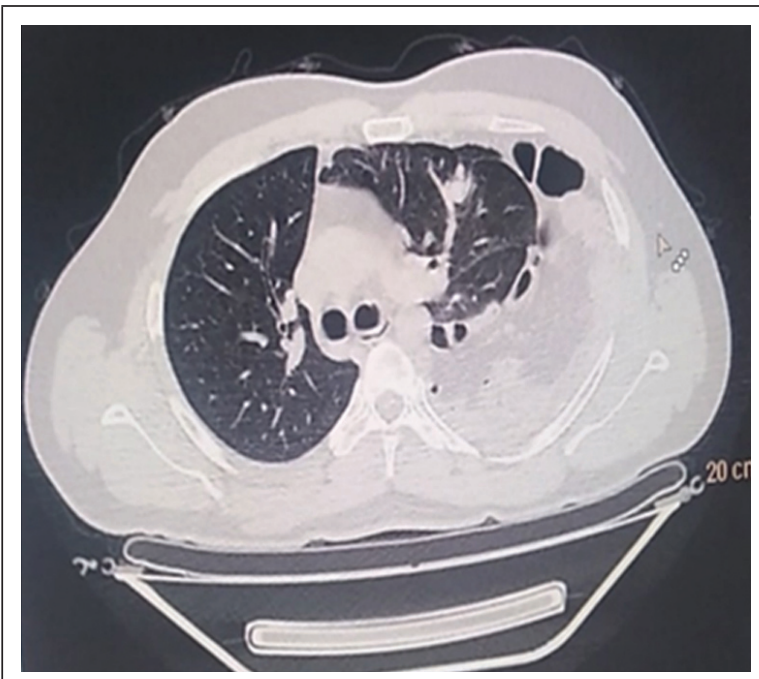

Figure 1: CT coronal view.

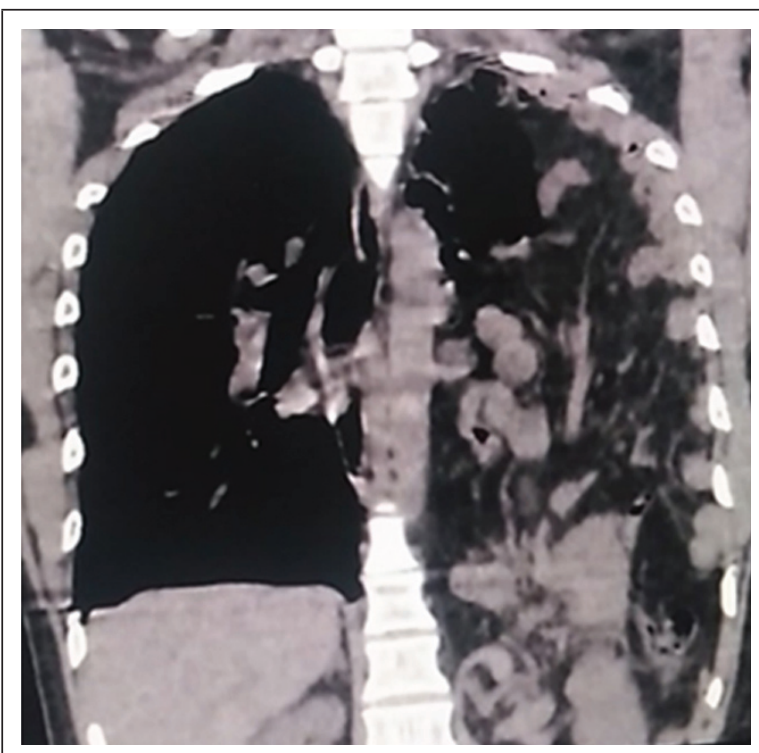

Figure 2: CT axial view.

Pulmonologist opinion was taken, and pulmonary function was optimized using bronchodilators and incentive spirometry in the preoperative period. 
After necessary preoperative investigations i.e., Hemoglobin $12.9 \mathrm{gm} \%$, total leucocyte count $8000 \mathrm{cell} /$ cumm, platelet count 2.16lakh/cumm, creatinine 0.48 , prothrombin time 12.4 and INR 1.3 , he was taken up for surgery.

The patient has undergone laparoscopic converted to laparotomy, in view of difficulty experienced in reducing the contents back into the abdominal cavity (Figure 3). There was not enough space in the abdominal cavity to proceed laparoscopically. Bleeding from omentum also contributed to making the decision to convert to open surgery.

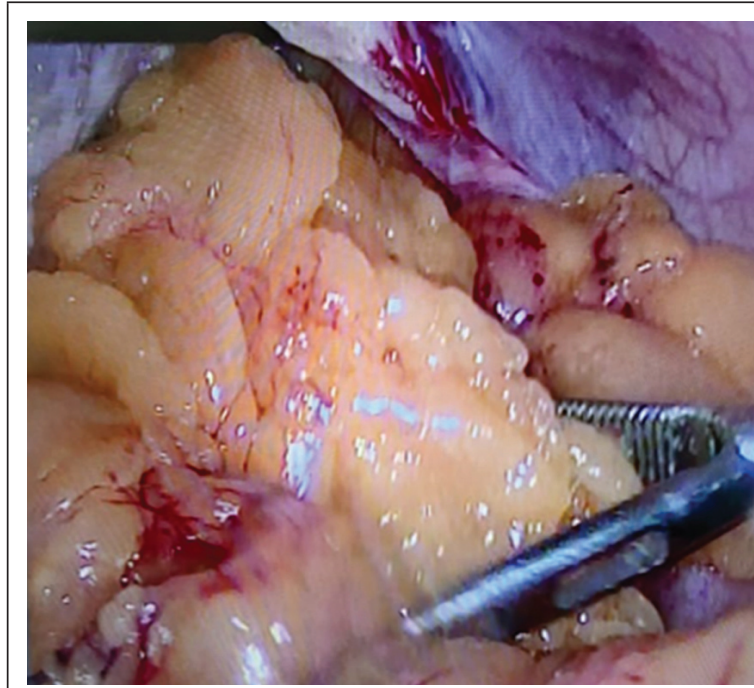

Figure 3: Intraoperative images of herniated.

After converting to laparotomy, it was noted that spleen, most of the colon, small bowel, and omentum herniated into the chest at costophrenic angle posteriorly. The size of diaphragmatic defect was about $8 \times 6 \mathrm{~cm}$ (Figure 4 ). The contents were reduced into the abdomen. Malrotation of gut was noted at this stage which is incomplete type of malrotation. The diaphragm was repaired using continuous ' 0 ' polyamide suture (Ethilon, Ethicon) (Figure 5). This was reinforced with a $15 \times 10 \mathrm{~cm}$ dual mesh (Parietex, Ethicon), secured with ' 0 ' polyamide suture (Figure 6). Caecum and ascending colon were placed in the right paracolic gutter and fixed using 2 ' 0 ' silk sutures. Intercostal drain (ICD) was placed in the left pleural cavity and connected to underwater seal drain. After 48-hour care in ICU, the patient was transferred to the surgical ward. He had prolonged post-operative ileus, high Ryle's tube output and required Oxygen supplementation. These were most likely due to increase in the intrabdominal pressure expected in this situation. With supportive treatment, he slowly improved, commenced on diet and weaned off oxygen.

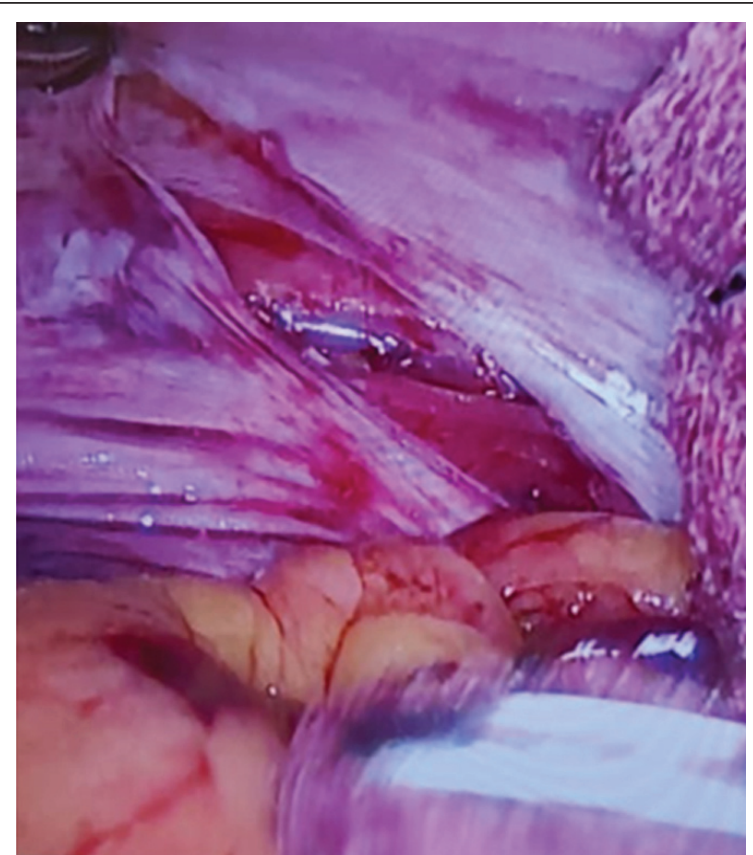

Figure 4: Defect in diaphragm omentum and small bowel.

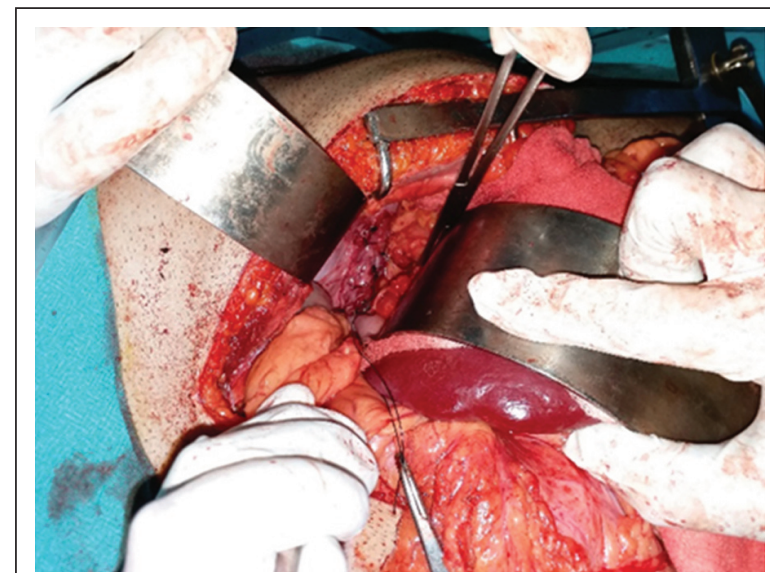

Figure 5: Defect closed with polyamide suture.

Chest X-ray in the post-operative period on sixth day (Figure 7), showed moderate amount of right pleural effusion, which was removed under ultrasound guidance. Fluid culture was sterile and biochemical analysis was within were in normal limits. ICD was removed on seventh postoperative day. The patient recovered well and was discharged from the hospital on tenth postoperative day. He was doing well on follow up, Abdominal wound healed 
well. Chest X-ray was normal (Figure 8) on sixteenth postoperative day.
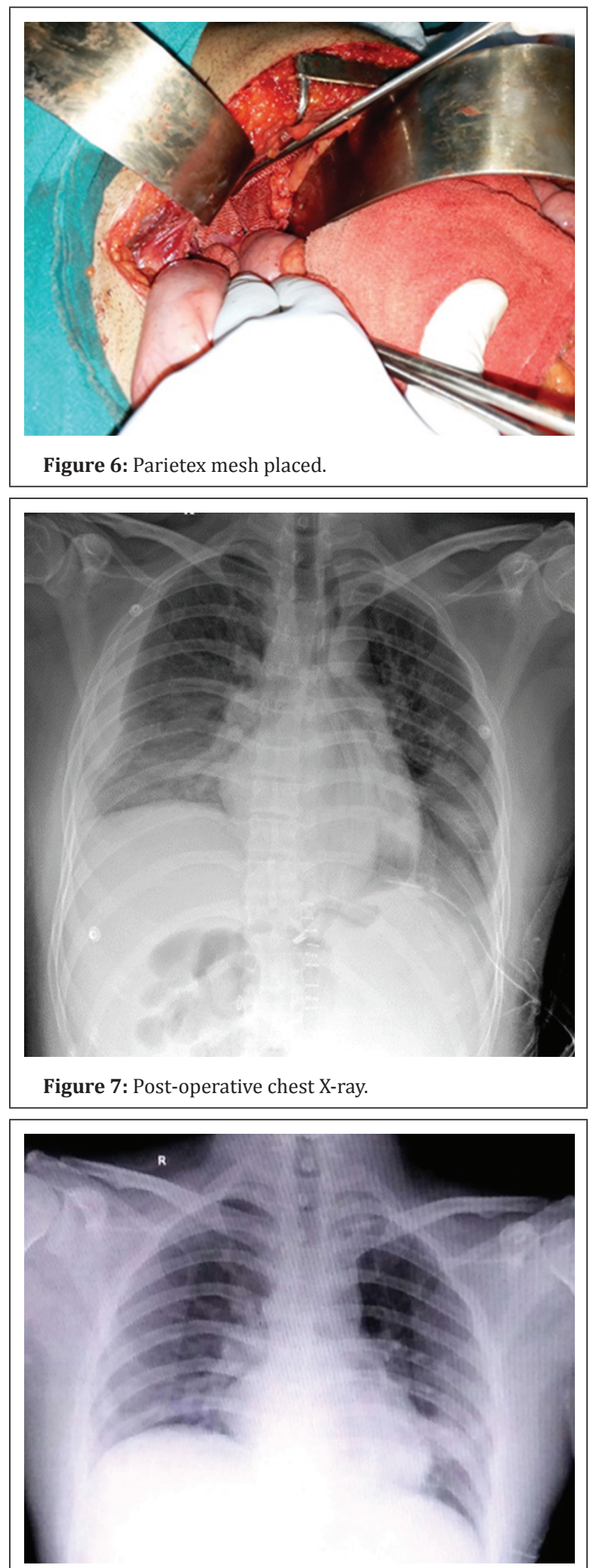

Figure 8: Chest X-ray on post-operative day-6.

\section{Discussion}

Bochdalek in 1834, first described a congenital defect of posterior-lateral part of diaphragm without hernia sac.Duetothecomplexity of congenital diaphragmatic hernia, the factors involved in the development of Bochdalek hernia are unknown; however, at present it is thought to be due to the lack of closure of pleural and peritoneal cavity due to disruption of molecular signalling during organogenesis around 9th to 10th weeks of gestation [7-10]. Bochdalek hernia is associated with chromosomal disorders (10-25\%) and other congenital defects (25-57\%). Diaphragmatic hernia beyond the neonatal period varies from $5 \%$ to $30 \%$. In adulthood, except for $\mathrm{CDH}$, there are different reasons such as trauma, phrenic nerve palsy, and delayed diagnosis of hiatus hernia for the development of diaphragmatic hernia. According to the results of an extensive study on patients with $\mathrm{CDH}$, a very small number of patients are diagnosed in adulthood with male to female ratio of 1:8. This study also concluded that it is very rare to present at 25 years of age, same age as our patient [6]. Chest X-ray is the most common imaging method to study diaphragm and heart. When the chest X-ray images are not diagnostic, spiral CT and or MRI are the next preferred imaging modalities, to acquire more information about the abnormal anatomy. CDH detection prior to surgery may be challenging because of rare incidence and difficulty in differentiating it from diaphragmatic eventration.

The patients with Bochdalek hernia, the diaphragm is defective, and the small intestine moves into thoracic cavity more than any other abdominal organs [2]. To prevent the serious complications of $\mathrm{CDH}$, surgical correction is advocated. Choosing the best treatment method for repairing $\mathrm{CDH}$ has become a challenge among the surgeons. Some of them support thoracotomy as the best treatment option because the chest is probably attached to hernia sac, while some others believe that laparotomy is better than thoracotomy for dealing with possible complications such as malrotation (incomplete rotation) in this case, obstruction, strangulation, and perforation of abdominal viscera. Furthermore, in recent years, minimal invasive surgery in the form of thoracoscopy and laparoscopy are replacing open methods to repair Bochdalek hernia. It is our practice to repair these defects using laparoscopic approach. 
Incomplete rotation is the most frequent type of malrotation of gut and occurs when there is a partial rotation of the duodenum and the right colon. The proximal portion of the midgut rotates $90^{\circ}$ and the distal portion rotates $180^{\circ}$, placing the duodenum on the right side of abdominal cavity and the cecum under the stomach. It results in a shorter distance between the TL and the cecum, and consequent narrowing of the mesentery root [11]. A thickened band of mesentery (Ladd's bands) can join the cecum to the duodenum and tighten the latter. If a large portion of intestine is left suspended from the posterior abdominal cavity with only one point of fixation, it is prone to torsion and formation of a volvulus which were not seen in this case. Incomplete or partial rotation of gut is associated with abdominal wall hernias (exomphalos major \& minor) followed by congenital diaphragmatic hernia which is seen in this case. Pre-operative MD CT abdomen would give a better way of diagnosing malrotation of gut if suspected which was not done in this case as there were no signs of obstruction.

\section{Conclusion}

A rare case of left-sided CDH with malrotation of gut (incomplete rotation) in an adult is presented. To our knowledge, this is one of the rarest cases in the literature to have the rare combination of $\mathrm{CDH}$ and malrotation of gut. Even with modern imaging methods, it is sometimes difficult to differentiate CDH from diaphragmatic eventration and to diagnose diaphragmatic hernias with malrotation. Definitive diagnosis of hernia and malrotation of gut before surgery may sometimes be difficult because of its infrequency and varied presentation. To improve clinical care and outcomes in adult CDH patients with intestinal malrotation, investigating appropriately and long-term follow-up are required in these patients, as with any other hernia repair to detect late recurrences and also signs of obstruction.

\section{Conflicts of interest}

Authors declare no conflicts of interest.

\section{References}

[1] Yamaguchi M, Kuwano H, Hashizume M, Sugio K, Sugimachi $\mathrm{K}$, et al. Thoracoscopic treatment of Bochdalek hernia in the adult: report of a case. Ann Thorac Cardiovasc Surg. 2002; 8(2):106-108.

[2] Goh BK, Teo MC, Chng SP, Soo KC. Right-sided Bochdalek's hernia in an adult. Am J Surg. 2007; 194(3):390-391.

[3] Dalencourt G, Katlic MR. Abdominal compartment syndrome after late repair of Bochdalek hernia. Ann Thorac Surg. 2006; 82(2):721-722.

[4] Fraser JD, Craft RO, Harold KL, Jaroszewski DE. Minimally invasive repair of a congenital right-sided diaphragmatic hernia in an adult. Surg Laparosc Endosc Percutan Tech. 2009; 19(1):e5-e7.

[5] Kanazawa A, Yoshioka Y, Inoi O, Murase J, Kinoshita H. Acute respiratory failure caused by an incarcerated right-sided adult bochdalek hernia: report of a case. Surg Today. 2002; 32(9):812-815.

[6] Luo HF, Lei T, Wang HJ, Tan G, Wang ZY. Non-traumatic diaphragmatic hernia of the liver in an adult: A case report. Hepatobiliary Pancreat Dis Int. 2007; 6(2):219-221.

[7] Bosenberg AT, Brown RA. Management of congenital diaphragmatic hernia. Curr Opin Anaesthesiol. 2008; 21(3):323-331.

[8] Liu LG, Xu YY, Mao YL, Sang XT, Yang ZY, et al. Adult congenital diaphragmatic hernia of the liver: a rare case report. Sci China Life Sci. 2010; 53(2):204-206.

[9] Slavotinek AM, Warmerdam B, Lin AE, Shaw GM. Populationbased analysis of left- and right-sided diaphragmatic hernias demonstrates different frequencies of selected additional anomalies. Am J Med Genet A. 2007; 143A(24):3127-3136.

[10] Gale ME. Bochdalekhernia: prevalence and CT characteristics. Radiology. 1985; 156(2):449-452.

[11] McVay MR, Kokoska ER, Jackson RJ, Smith SD. Jack Barney Award. The changing spectrum of intestinal malrotation: diagnosis and management. Am J Surg. 2007, 194(6):712717.- 